\title{
Consistency analysis of a nonbirefringent Lorentz-violating planar model
}

\author{
Rodolfo Casana, Manoel M. Ferreira Jr, Roemir P. M. Moreira \\ Departamento de Física, Universidade Federal do Maranhão (UFMA), \\ Campus Universitário do Bacanga, São Luís - MA, 65085-580, Brazil.
}

\begin{abstract}
In this work analyze the physical consistency of a nonbirefringent Lorentz-violating planar model via the analysis of the pole structure of its Feynman's propagators. The nonbirefringent planar model, obtained from the dimensional reduction of the CPT-even gauge sector of the standard model extension, is composed of a gauge and a scalar fields, being affected by Lorentz-violating (LIV) coefficients encoded in the symmetric tensor $\kappa_{\mu \nu}$. The propagator of the gauge field is explicitly evaluated and expressed in terms of linear independent symmetric tensors, presenting only one physical mode. The same holds for the scalar propagator. A consistency analysis is performed based on the poles of the propagators. The isotropic parity-even sector is stable, causal and unitary mode for $0 \leq \kappa_{00}<1$. On the other hand, the anisotropic sector is stable and unitary but in general noncausal. Finally, it is shown that this planar model interacting with a $\lambda|\varphi|^{4}-$ Higgs field supports compactlike vortex configurations.
\end{abstract}

PACS numbers: $11.30 . \mathrm{Cp}, 12.60 .-\mathrm{i}, 11.55 . \mathrm{Fv}$

\section{INTRODUCTION}

The gauge sector of the of the Standard Model Extension (SME) [1, 2] embraces a CPT-odd [3] and a CPT-even [4 -7$]$ subsectors, which have been examined in several aspects concerning supersymmetry [8], vacuum Cherenkov radiation emission [9, 10], radiative corrections [11], Casimir effect [12], anisotropies of the Cosmic Microwave Background Radiation [13], between other interesting issues [14]. Some work was devoted to examine consistency aspects of the CPT-odd subsector [15, 16] and of the CPT-even one [17, 18].

The CPT-even gauge sector of the SME is represented by the CPT-even tensor Lagrangian

$$
\mathcal{L}_{(1+3)}=-\frac{1}{4} F_{\hat{\mu} \hat{\nu}} F^{\hat{\mu} \hat{\nu}}-\frac{1}{4}\left(K_{F}\right)_{\hat{\mu} \hat{\nu} \hat{\lambda} \hat{\kappa}} F^{\hat{\mu} \hat{\nu}} F^{\hat{\lambda} \hat{\kappa}},
$$

with $\left(K_{F}\right)_{\alpha \beta \mu \nu}$ being the Lorentz-violating tensor composed of 19 coefficients, with nine nonbirefringent and ten birefringent ones, endowed with the symmetries of the Riemann tensor, and a double null trace, $\left(K_{F}\right)^{\hat{\mu} \hat{\nu}} \hat{\mu} \hat{\nu}=0$. The effects of this CPT-even electrodynamics on fermion-fermion interaction was considered in Refs. 19 23].

A Lorentz-violating and CPT-even model in $(1+2)$-dimensions [24] was first derived by means of the dimensional reduction of the CPT-even Lagrangian (1), leading to the following planar Lagrangian

$$
\mathcal{L}_{(1+2)}=-\frac{1}{4} F_{\mu \nu} F^{\mu \nu}-\frac{1}{4} Z_{\mu \nu \lambda \kappa} F^{\mu \nu} F^{\lambda \kappa}+\frac{1}{2} \partial_{\mu} \phi \partial^{\mu} \phi-C_{\mu \lambda} \partial^{\mu} \phi \partial^{\lambda} \phi+T_{\mu \lambda \kappa} \partial^{\mu} \phi F^{\lambda \kappa},
$$

where $Z_{\mu \nu \lambda \kappa}, C_{\mu \lambda}, T_{\mu \lambda \kappa}$ are Lorentz-violating tensors which present together 19 components and affect the electromagnetic and scalar sectors. Further investigations concerning Lorentz violation in $(1+2)$ dimension focused on the nonbirefringent CPT-even electrodynamics of the SME, governed by the Lagrangian

$$
\mathcal{L}_{(1+3)}=-\frac{1}{4} F_{\hat{\mu} \hat{\nu}} F^{\hat{\mu} \hat{\nu}}-\frac{1}{2} \kappa_{\hat{\nu} \hat{\rho}} F_{\hat{\lambda}}^{\hat{\nu}} F^{\hat{\lambda} \hat{\rho}},
$$

whose components are parametrized in the symmetric and traceless tensor, $\kappa_{\hat{\nu} \hat{\rho}}$. Its dimensional reduction provided the following planar Lagrangian [25]:

$$
\mathcal{L}_{1+2}=-\frac{1}{4} F_{\mu \nu} F^{\mu \nu}-\frac{1}{2} \kappa_{\nu \rho} F_{\lambda}{ }^{\nu} F^{\lambda \rho}+\frac{1}{2}[1-\eta] \partial_{\mu} \phi \partial^{\mu} \phi+\frac{1}{2} \kappa_{\nu \rho} \partial^{\nu} \phi \partial^{\rho} \phi+S_{\nu} F^{\nu \lambda} \partial_{\lambda} \phi,
$$

influenced by 9 Lorentz-violating coefficients contained in the tensors $\kappa_{\nu \rho}, S_{\nu}$. This planar model is composed of parity-odd and parity-even components, allowing potential applications in general planar systems 31. Both planar Lagrangian densities (2) and (4) present scalar sectors endowed with a noncanonical kinetic term, which has been used 
to study topological defects in $(1+1)$ dimensions [26], acoustic black holes with Lorentz-violation in $(1+2)$ dimensions [27] and also the Bose-Einstein condensation of a bosonic ideal gas [28].

In this work, we finalize the investigation concerning consistency aspects of the planar model described by Lagrangian (44), whose properties were first analyzed in Ref. [25]. With this purpose, we exactly evaluate the Feynman's propagators for the electromagnetic and scalar sectors. Such calculation, for the gauge field, is performed by means of a closed set of tensors (non necessarily linearly independents), previously defined in Ref. 17]. After expressing the propagator in terms of linearly independent tensors, we proceed writing the dispersion relations for this planar theory and comparing them with the relations of Ref. [25]. The physical dispersion relations are used to examine the causality, stability and unitarity. It is shown that this planar theory is endowed with causality illness, being stable and unitary for some known range of parameters. As a clear physical motivation to study this kind of planar model, we show that it constitutes a suitable framework for studying nonconventional vortex solutions. Indeed, it allows the existence of compactlike uncharged BPS vortex configurations, whenever coupled with the suitable a $\lambda|\varphi|^{4}-$ Higgs potential.

This work is outlined as follows. In Sec. II, we explicitly carry out the Feynman's propagators using a suitable parametrization for the LIV tensor. In Sec. III, we analyze by using the dispersion relations, the stability, causality and unitarity issues concerning this theory. In Sec. IV, we discuss the existence of BPS vortex configurations. In Sec. V, we present our Conclusions.

\section{FEYNMAN PROPAGATOR FOR THE PLANAR THEORY}

It we disregard the mixing term $\left(S_{\mu}=0\right)$ in the Lagrangian (4), the gauge and scalar sectors turns out uncoupled, being governed by

$$
\mathcal{L}_{1+2}=-\frac{1}{4} F_{\mu \nu} F^{\mu \nu}-\frac{1}{2} \kappa_{\nu \rho} F_{\lambda}{ }^{\nu} F^{\lambda \rho}-\frac{1}{2 \xi}\left(\partial_{\mu} A^{\mu}\right)^{2}+\frac{1}{2}[1-\eta] \partial_{\mu} \phi \partial^{\mu} \phi+\frac{1}{2} \kappa_{\nu \rho} \partial^{\nu} \phi \partial^{\rho} \phi,
$$

where we have introduced the gauge-fixing term $\left(\partial_{\mu} A^{\mu}\right)^{2} / 2 \xi$, and the parameter $\eta$ given by

$$
\eta=\kappa_{00}-\operatorname{tr}\left(\kappa_{i j}\right) \text {. }
$$

Such Lagrangian can be written as

$$
\mathcal{L}_{1+2}=\frac{1}{2} A_{\mu}\left[D^{\mu \nu}\right] A_{\nu}+\frac{1}{2} \phi[\boxminus] \phi
$$

where the operator $D^{\mu \nu}$ defines the kinetic term of the gauge field

$$
D^{\mu \nu}=\left(\square+\kappa^{\alpha \beta} \partial_{\alpha} \partial_{\beta}\right) g^{\mu \nu}+\lambda \partial^{\mu} \partial^{\nu}+\square \kappa^{\mu \nu}-\kappa^{\mu \alpha} \partial_{\alpha} \partial^{\nu}-\kappa^{\nu \alpha} \partial_{\alpha} \partial^{\mu},
$$

where $\lambda=\xi^{-1}-1$, and $\square$ the scalar kinetic term

$$
\square=-(1-\eta) \square-\kappa_{\rho \sigma} \partial^{\rho} \partial^{\sigma} .
$$

In this situation, we initiate evaluating the Feynman propagator for the gauge sector, which fulfills the relation

$$
D^{\mu \beta} \Delta_{\beta \nu}(x-y)=\delta^{\mu}{ }_{\nu} \delta(x-y) .
$$

The gauge propagator is defined as $i \Delta_{\mu \nu}(x-y)=\left\langle 0\left|T A_{\mu}(x) A_{\nu}(y)\right| 0\right\rangle$, where $\Delta_{\mu \nu}(x-y)$ is the Green's function of the operator $D^{\mu \nu}$ that appears in Eq. (8). Working in the Fourier representation, we get

$$
\widetilde{D}^{\mu \nu}(p)=-\left(p^{2}+\kappa^{\alpha \beta} p_{\alpha} p_{\beta}\right) g^{\mu \nu}-\lambda p^{\mu} p^{\nu}-p^{2} \kappa^{\mu \nu}+\kappa^{\mu \alpha} p_{\alpha} p^{\nu}+\kappa^{\nu \alpha} p_{\alpha} p^{\mu} .
$$

is a suitable way for inverting this tensor, which amounts at finding a tensor $\tilde{\Delta}_{\mu \nu}$ that fulfills $\tilde{D}^{\mu \beta} \tilde{\Delta}_{\beta \nu}=\delta^{\mu}{ }_{\nu}$. Thus, we use the following parametrization for a symmetric tensor,

$$
\kappa^{\mu \nu}=\frac{1}{2}\left(A^{\mu} B^{\nu}+A^{\nu} B^{\mu}\right),
$$


with nonnull trace, $\kappa^{\alpha}{ }_{\alpha}=A \cdot B \neq 0$, and $A^{\mu}, B^{\nu}$ are two arbitrary three-vectors which comprise the Lorentz-violating coefficients. Moreover, we have

$$
\kappa^{00}=A^{0} B^{0}, \kappa^{0 i}=\frac{1}{2}\left(A^{0} B^{i}+A^{i} B^{0}\right), \kappa^{i j}=\frac{1}{2}\left(A^{i} B^{j}+A^{j} B^{i}\right),
$$

here, $\kappa^{00}$ is the isotropic parity-even parameter, $\kappa^{0 i}$ and $\kappa^{i j}$ are the anisotropic LIV coefficients retaining the others parity-even and parity-odd ones. Replacing the parametrization (12) in Eq.(11), we have:

$$
\begin{aligned}
\widetilde{D}^{\mu \nu} & =-\left[p^{2}+(p \cdot A)(p \cdot B)\right] g^{\mu \nu}-\lambda p^{\mu} p^{\nu}-\frac{1}{2} p^{2}\left(A^{\mu} B^{\nu}+A^{\nu} B^{\mu}\right) \\
& +\frac{1}{2}(p \cdot A)\left(p^{\mu} B^{\nu}+p^{\nu} B^{\mu}\right)+\frac{1}{2}(p \cdot B)\left(p^{\mu} A^{\nu}+p^{\nu} A^{\mu}\right) .
\end{aligned}
$$

In order to compute the inverse of $\widetilde{D}^{\mu \nu}$, we use the closed algebra satisfied by the following tensors:

$$
\Theta_{\mu \nu}, \omega_{\mu \nu}, A_{\mu} B_{\nu}, A_{\nu} B_{\mu}, p_{\mu} A_{\nu}, p_{\nu} A_{\mu}, p_{\mu} B_{\nu}, p_{\nu} B_{\mu}, A_{\mu} A_{\nu}, B_{\mu} B_{\nu}
$$

which is presented in the Tables of Ref. [17]. Here, $\Theta_{\mu \nu}=g_{\mu \nu}-\omega_{\mu \nu}$ and $\omega_{\mu \nu}=p_{\mu} p_{\nu} / p^{2}$ are the transverse and longitudinal projectors, respectively. Due to the tensor $\widetilde{D}^{\mu \nu}$ is symmetric in the Lorentz indices and under the exchange $A \leftrightarrow B$, the gauge field propagator is expressed as

$$
\begin{aligned}
\widetilde{\Delta}_{\mu \nu}(p) & =\alpha_{1} \Theta_{\mu \nu}+\alpha_{2} \omega_{\mu \nu}+\alpha_{3} A_{\mu} A_{\nu}+\alpha_{4}\left(A_{\mu} B_{\nu}+A_{\nu} B_{\mu}\right) \\
& +\alpha_{5}\left(p_{\mu} A_{\nu}+p_{\nu} A_{\mu}\right)+\alpha_{6} B_{\mu} B_{\nu}+\alpha_{7}\left(p_{\mu} B_{\nu}+p_{\nu} B_{\mu}\right)
\end{aligned}
$$

with the coefficients $\alpha_{i}$ given as

$$
\begin{aligned}
& \alpha_{1}=-\frac{1}{p^{2}+(A \cdot p)(B \cdot p)} \quad, \quad \alpha_{3}=\frac{(B \cdot p)^{2}-p^{2} B^{2}}{4 \otimes\left[p^{2}+(A \cdot p)(B \cdot p)\right]} \\
& \alpha_{2}=\frac{2(A \cdot B)(A \cdot p)(B \cdot p)-A^{2}(B \cdot p)^{2}-B^{2}(A \cdot p)^{2}}{4 \bigotimes\left[p^{2}+(A \cdot p)(B \cdot p)\right]}+\frac{(A \cdot p)(B \cdot p)}{p^{2} \bigotimes}-\frac{\xi}{p^{2}} \\
& \alpha_{4}=\frac{p^{2}[2+(A \cdot B)]+(A \cdot p)(B \cdot p)}{4 \bigotimes\left[p^{2}+(A \cdot p)(B \cdot p)\right]}, \quad \alpha_{6}=\frac{(A \cdot p)^{2}-p^{2} A^{2}}{4 \bigotimes\left[p^{2}+(A \cdot p)(B \cdot p)\right]} \\
& \alpha_{5}=-\frac{p^{2}\left[2(B \cdot p)+(A \cdot B)(B \cdot p)-B^{2}(A \cdot p)\right]+2(A \cdot p)(B \cdot p)^{2}}{4 p^{2} \bigotimes\left[p^{2}+(A \cdot p)(B \cdot p)\right]} \\
& \alpha_{7}=-\frac{p^{2}\left[2(A \cdot p)+(A \cdot B)(A \cdot p)-A^{2}(B \cdot p)\right]+2(B \cdot p)(A \cdot p)^{2}}{4 p^{2} \bigotimes\left[p^{2}+(A \cdot p)(B \cdot p)\right]}
\end{aligned}
$$

where $A^{2}=A_{\mu} A^{\mu}, B^{2}=B_{\mu} B^{\mu}$, and the denominator element $\otimes$ is

$$
\begin{gathered}
\bigotimes(p)=f(A, B) p^{2}+(A \cdot p)(B \cdot p)+\frac{1}{2}(A \cdot B)(A \cdot p)(B \cdot p)+\frac{1}{4} B^{2}(A \cdot p)^{2}+\frac{1}{4} A^{2}(B \cdot p)^{2}, \\
f(A, B)=1+(A \cdot B)+\frac{1}{4}(A \cdot B)^{2}-\frac{1}{4} B^{2} A^{2} .
\end{gathered}
$$

At first sight, the gauge field propagator presents two simple poles in $\bigotimes=0$ and $p^{2}+(A \cdot p)(B \cdot p)=0$, and a double pole in $p^{2}=0$. However, as it will be shown below, the gauge field presents only a unique physical pole, $\otimes=0$, that is related to its physical dispersion relation. The second pole, $p^{2}+(A \cdot p)(B \cdot p)=0$, appears due the fact the gauge propagator (16) is expressed in terms of seven symmetric and linearly dependent tensors arranged from the ten original tensors given in Eq. (15). It is well known that any second order symmetric tensor in $(2+1)$-D can be 
written in terms of only six symmetric and linearly independent (L.I.) ones. By choosing the set of L.I. tensors as being $\left\{g^{\mu \nu}, \omega_{\mu \nu}, A_{\mu} A_{\nu}, A_{\mu} B_{\nu}+A_{\nu} B_{\mu}, p_{\mu} A_{\nu}+p_{\nu} A_{\mu}, p_{\mu} B_{\nu}+p_{\nu} B_{\mu}\right\}$, we can express the tensor $B^{\mu} B^{\nu}$, in terms of this L.I. set, in the form

$$
B^{\mu} B^{\nu}=\beta_{1} g^{\mu \nu}+\beta_{2} p^{2} \omega^{\mu \nu}+\beta_{3} A^{\mu} A^{\nu}+\beta_{4} \frac{A^{\mu} B^{\nu}+A^{\nu} B^{\mu}}{2}+\beta_{5} \frac{A^{\mu} p^{\nu}+A^{\nu} p^{\mu}}{2}+\beta_{6} \frac{B^{\mu} p^{\nu}+B^{\nu} p^{\mu}}{2}
$$

where

$$
\begin{aligned}
& \beta_{1}=-\frac{p^{2}\left[A^{2} B^{2}-(A \cdot B)^{2}\right]+2(A \cdot p)(A \cdot B)(B \cdot p)-A^{2}(B \cdot p)^{2}-B^{2}(A \cdot p)^{2}}{(A \cdot p)^{2}-A^{2} p^{2}}, \\
& \beta_{2}=-\frac{(A \cdot B)^{2}-A^{2} B^{2}}{(A \cdot p)^{2}-A^{2} p^{2}}, \quad \beta_{3}=-\frac{(B \cdot p)^{2}-B^{2} p^{2}}{(A \cdot p)^{2}-A^{2} p^{2}}, \quad \beta_{4}=-\frac{2(A \cdot B) p^{2}-2(p \cdot A)(p \cdot B)}{(A \cdot p)^{2}-A^{2} p^{2}}, \\
& \beta_{5}=-\frac{2(p \cdot A) B^{2}-2(A \cdot B)(p \cdot B)}{(A \cdot p)^{2}-A^{2} p^{2}}, \quad \beta_{6}=-\frac{2(p \cdot B) A^{2}-2(A \cdot B)(p \cdot A)}{(A \cdot p)^{2}-A^{2} p^{2}},
\end{aligned}
$$

By replacing the expression for $B^{\mu} B^{\nu}$ in Eq. (16), and after some considerable algebraic effort, we obtain:

$$
\begin{aligned}
\widetilde{\Delta}_{\mu \nu}(p) & =-\frac{1+(A \cdot B)}{\bigotimes} \Theta_{\mu \nu}+\left[\frac{(A \cdot p)(B \cdot p)}{p^{2} \bigotimes}-\frac{\xi}{p^{2}}\right] \omega_{\mu \nu}+\frac{A_{\mu} B_{\nu}+A_{\nu} B_{\mu}}{2 \bigotimes} \\
& -(B \cdot p) \frac{A_{\mu} p_{\nu}+A_{\nu} p_{\mu}}{2 p^{2} \bigotimes}-(A \cdot p) \frac{B_{\mu} p_{\nu}+B_{\nu} p_{\mu}}{2 p^{2} \bigotimes} .
\end{aligned}
$$

In this form, $\widetilde{\Delta}_{\mu \nu}$ is expressed in terms only five L.I. symmetric tensors, involving just eight of the original tensor set (15). The equation above now can also be expressed in terms of the tensor $\kappa^{\mu \nu}$ as

$$
\widetilde{\Delta}_{\mu \nu}(p)=-\frac{\left(1+\kappa_{\alpha}^{\alpha}\right)}{\bigotimes} \Theta_{\mu \nu}+\left[\frac{\kappa^{\rho \sigma} p_{\rho} p_{\sigma}}{p^{2} \otimes}-\frac{\xi}{p^{2}}\right] \omega_{\mu \nu}+\frac{\kappa_{\mu \nu}}{\bigotimes}-\frac{p_{\mu} \kappa_{\nu \sigma} p^{\sigma}+p_{\nu} \kappa_{\mu \sigma} p^{\sigma}}{p^{2} \bigotimes}
$$

and

$$
\nabla=\left[1+\kappa_{\alpha}^{\alpha}+\frac{1}{2}\left(\kappa_{\alpha}^{\alpha}\right)^{2}-\frac{1}{2}\left(\kappa^{\alpha \beta} \kappa_{\alpha \beta}\right)\right] p^{2}+\kappa^{\mu \nu} p_{\mu} p_{\nu}+\kappa^{\mu \alpha} \kappa_{\alpha}{ }^{\nu} p_{\mu} p_{\nu}
$$

It can be explicitly verified that the expression (29) is the inverse of the tensor $\widetilde{D}^{\mu \nu}(p)$ appearing in (11), for any symmetric $\kappa^{\mu \nu}$. Thus, the propagator in momentum space reads

$$
\left\langle 0\left|T A_{\mu}(p) A_{\nu}(-p)\right| 0\right\rangle=-\frac{i}{\bigotimes}\left[\left(1+\kappa_{\alpha}^{\alpha}\right) \Theta_{\mu \nu}-\left(\frac{\kappa^{\rho \sigma} p_{\rho} p_{\sigma}}{p^{2}}-\xi \frac{\bigotimes}{p^{2}}\right) \omega_{\mu \nu}-\kappa_{\mu \nu}+\frac{p_{\mu} \kappa_{\nu \sigma} p^{\sigma}+p_{\nu} \kappa_{\mu \sigma} p^{\sigma}}{p^{2}}\right]
$$

We can observe that the propagator presents a simple pole in $\otimes=0$ and a double pole in $p^{2}=0$. As it will be shown, the pole $\otimes=0$ is the one that provides physical dispersion relations, while the pole $p^{2}=0$ is unphysical.

One should evaluate the Feynman propagator for the scalar sector as well, which in momentum space fulfills

$$
\langle 0|T \phi(p) \phi(-p)| 0\rangle=i \tilde{\Delta}(p)
$$

where $\tilde{\Delta}(p)$ satisfies $\tilde{\bullet} \tilde{\Delta}=1$, and $\tilde{\bullet}$ is the momentum representation of the scalar kinetic term defined in (9) $\tilde{\Delta}^{-1}=(1-\eta) p^{2}+\kappa^{\rho \sigma} p_{\rho} p_{\sigma}$. Thus, the scalar propagator reads

$$
\langle 0|T \phi(p) \phi(-p)| 0\rangle=\frac{i}{(1-\eta) p^{2}+\kappa^{\rho \sigma} p_{\rho} p_{\sigma}} .
$$

Note that it has a simple pole in $\tilde{\dot{v}}=0$. 


\section{DISPERSION RELATIONS}

The dispersion relations for the gauge sector are read off from the physical poles of the gauge propagator, that is, $\bigotimes=0$, or explicitly

$$
\left[1+\kappa_{\alpha}^{\alpha}+\frac{1}{2}\left(\kappa_{\alpha}^{\alpha}\right)^{2}-\frac{1}{2}\left(\kappa^{\alpha \beta} \kappa_{\alpha \beta}\right)\right] p^{2}+\kappa^{\mu \nu} p_{\mu} p_{\nu}+\kappa^{\mu \alpha} \kappa_{\alpha}{ }^{\nu} p_{\mu} p_{\nu}=0
$$

while the dispersion relation for the scalar sector is extracted from the scalar propagator,

$$
(1-\eta) p^{2}+\kappa^{\rho \sigma} p_{\rho} p_{\sigma}=0
$$

These dispersion relations are the same ones that were explicitly computed in Ref. [25] starting from the vacuumvacuum amplitude transition. With both relations in hands we can analyze the energy stability, causality and unitarity of this theory.

\section{A. Stability}

We initiate analyzing the isotropic parity-even sector characterized by the parameter $\kappa_{00}$. The expression (34) yields only a unique dispersion relation for the gauge field,

$$
p_{0}= \pm \frac{|\mathbf{p}|}{\sqrt{1+\kappa_{00}}}
$$

which stands for positive-energy modes being compatible with energy stability for $\kappa_{00}>-1$. For the same nonnull LIV coefficient, the scalar dispersion relation Eq. (35) provides

$$
p_{0}= \pm|\mathbf{p}| \sqrt{1-\kappa_{00}}
$$

which stands for positive-energy modes being compatible with energy stability for $\kappa_{00}<1$. Concerning the isotropic parameter, therefore, the entire model will be stable for $\left|\kappa_{00}\right|<1$.

Taking now as nonnull only the anisotropic parameter $\kappa_{0 i}$, the dispersion relation (34) for the gauge field is

$$
p_{0}=\kappa_{0 i} p_{i} \pm|\mathbf{p}| \sqrt{1+\left(\kappa_{0 i}\right)^{2}}
$$

while for the scalar degree of freedom, Eq. (35) yields

$$
p_{0}=\kappa_{0 i} p_{i} \pm|\mathbf{p}| \sqrt{1+\frac{\left(\kappa_{0 i} p_{i}\right)^{2}}{\mathbf{p}^{2}}} .
$$

These dispersion relations represent modes compatible with energy stability for any value of the LIV parameter $\kappa_{0 i}$.

For nonnull $\kappa_{i j}$, the dispersion relation (34) for the gauge degree of freedom takes the form

$$
p_{0}= \pm N_{0}|\mathbf{p}| \sqrt{1-\kappa_{i j} p_{i} p_{j} / \mathbf{p}^{2}}
$$

with $N_{0}=\sqrt{\left(1-\operatorname{tr}\left(\kappa_{i j}\right)\right) /\left(1-\operatorname{tr}\left(\kappa_{i j}\right)+\operatorname{det}\left(\kappa_{i j}\right)\right)}$. From Eq. (35), the scalar field supports the dispersion relation

$$
p_{0}= \pm|\mathbf{p}| \sqrt{1+\left[1+\operatorname{tr}\left(\kappa_{i j}\right)\right]^{-2} \kappa_{i j} p_{i} p_{j} / \mathbf{p}^{2}}
$$

These dispersion relations give modes supporting energy stability for sufficiently small values of the parameter $\kappa_{i j}$. 


\section{B. Causality analysis}

The poles of the Feynman propagator can be properly used to analyze the causality of the associated theory [29]. In order to have a causal mode (preventing the existence of tachyons), one must have $p^{2} \geq 0$. A mode confident criterium for defining causality is the evaluation of the group and front velocities $\left(u_{g}=d p_{0} / d|\mathbf{p}|, u_{\text {front }}=\lim _{|\mathbf{p}| \rightarrow \infty} u_{\text {phase }}\right)$, so that causality is ensured if $u_{g}, u_{\text {front }} \leq 1$. In Refs. [17], the causality of the original four-dimensional CPT-even model was examined in its parity-odd and parity-even subsectors, revealing the existence of noncausal modes.

As for the isotropic sector, the dispersion relations (36) and (37) yield the group velocities

$$
u_{g}=\left(1+\kappa_{00}\right)^{-1 / 2}, u_{g}=\left(1-\kappa_{00}\right)^{1 / 2},
$$

which lead to causal modes once the condition $0 \leq \kappa_{00} \leq 1$ is obeyed (criterium for having $u_{g}, u_{\text {front }} \leq 1$ ), otherwise the causality is violated. As for the anisotropic modes ruled by $\kappa_{0 i}$, we consider the dispersion relations (38) and (39), whose respective group velocities are

$$
u_{g}=\sqrt{1+\left(\kappa_{0 i}\right)^{2}} \pm\left|\kappa_{0 i}\right| \cos \theta_{i}, u_{g}=\sqrt{1+\left|\kappa_{0 i}\right|^{2} \cos ^{2} \theta} \pm\left|\kappa_{0 i}\right| \cos \theta_{i} .
$$

where $\cos \theta_{i}=p_{i} /|\mathbf{p}|$. The anisotropic modes associated with $\kappa_{i j}$ are described by the dispersion relations (40) and (41) which provide the following group velocities

$$
u_{g}=N_{0} \sqrt{1-\kappa_{i j} p_{i} p_{j} / \mathbf{p}^{2}}, \quad u_{g}=\sqrt{1+\left[1+\operatorname{tr}\left(\kappa_{i j}\right)\right]^{-2} \kappa_{i j} p_{i} p_{j} / \mathbf{p}^{2}} .
$$

As the group velocities (43) (44) may be larger than 1 for some specific configurations of $\kappa_{0 i}, \kappa_{i j}$, we conclude that these anisotropic modes are in general noncausal.

\section{Unitarity analysis}

The unitarity analysis of this model at tree-level is here carried out through the saturation of the propagators with external currents [30], which must be implemented by means of the saturated propagator $(S P)$, a scalar quantity given as follows:

$$
S P=J^{* \mu} \operatorname{Res}\left(i \Delta_{\mu \nu}\right) J^{\nu},
$$

where $\operatorname{Res}\left(i \Delta_{\mu \nu}\right)$ is the matrix residue evaluated at the pole of the propagator. The gauge current $\left(J^{\mu}\right)$ satisfies the conservation law $\left(\partial_{\mu} J^{\mu}=0\right)$, which in momentum space is read as $p_{\mu} J^{\mu}=0$. In accordance with this method, the unitarity analysis is assured whenever the imaginary part of the saturation $S P$ (at the poles of the propagator) is positive-definite. A way to carry out the saturation consists of determining the residue of the propagator matrix, evaluated at its own poles. Such analysis can state that a mode is physical or nonphysical (whenever the saturation is positive or nonnegative), leading to a result which should be reconciliated with the existence of only a physical mode.

From the propagator given in (31) the residue in the pole $\nabla(p)=0$ is

$$
\operatorname{Res}\left(i \tilde{\Delta}_{\mu \nu}\right)=-\frac{g_{\mu \nu}\left(1+\kappa_{\alpha}^{\alpha}\right)}{\varrho}+\left[\frac{\varrho \kappa^{\rho \sigma} p_{\rho} p_{\sigma}}{\left(\Sigma-\mathbf{p}^{2} \varrho\right)^{2}}-\frac{\left(1+\kappa_{\alpha}^{\alpha}\right)}{\Sigma-\mathbf{p}^{2} \varrho}\right] p_{\mu} p_{\nu}+\frac{\kappa_{\mu \nu}}{\varrho}-\frac{p_{\mu} \kappa_{\nu \sigma} p^{\sigma}+p_{\nu} \kappa_{\mu \sigma} p^{\sigma}}{\Sigma-\mathbf{p}^{2} \varrho}
$$

where

$$
\begin{aligned}
& \Sigma=2 p_{0}\left[\left(1+\kappa_{00}\right)\left(\kappa_{0 i} p_{i}\right)-\left(\kappa_{0 i} \kappa_{i j} p_{j}\right)\right]-\left[1-\operatorname{tr}\left(\kappa_{i j}\right)\right]\left[\left(\kappa_{i j} p_{i} p_{j}\right)-\mathbf{p}^{2}\left(1+\kappa_{00}\right)\right]+\left(\epsilon_{i j} \kappa_{0 i} p_{j}\right)^{2}, \\
& \varrho=\left(1+\kappa_{00}\right)\left[1+\kappa_{00}-\operatorname{tr}\left(\kappa_{i j}\right)\right]+\operatorname{det}\left(\kappa_{i j}\right) .
\end{aligned}
$$

Then the saturated residue of the gauge propagator (taking into account the current conservation) is

$$
S P=i \frac{J^{\mu} \kappa_{\mu \nu} J^{\nu}-J^{2}\left(1+\kappa_{\alpha}^{\alpha}\right)}{\varrho}
$$


We will investigate the unitarity for three situations: (i) the isotropic coefficient, $\kappa_{00}$, (ii) the anisotropic coefficients, $\kappa_{0 i}$, (iii) the anisotropic coefficients, $\kappa_{i j}$.

We consider first the isotropic configuration, represented by $\kappa_{00}$. In this case, $\varrho=\left(1+\kappa_{00}\right)^{2}$ and the saturation is

$$
S P=i \frac{(\mathbf{p} \times \mathbf{J})^{2}}{\left(1+\kappa_{00}\right) \mathbf{p}^{2}},
$$

which will imply a positive-definite imaginary part whenever $\kappa_{00}>-1$. The isotropic parity-even sector gives an stable and unitary for $\left|\kappa_{00}\right|<1$,

We consider now the anisotropic configuration associated with the coefficient $\kappa_{0 i}$. In this case, $\varrho=1$ and the saturation is

$$
S P=i\left[\mathbf{J}^{2}-\left(J_{0}\right)^{2}-2 \kappa_{0 i} J_{0} J_{i}\right]
$$

The saturation can be written as

$$
\mathbf{J}^{2}-\left(J_{0}\right)^{2}-2 \kappa_{0 i} J_{0} J_{i}=\frac{\mathcal{J}}{\left(\boldsymbol{\Lambda} \cdot \mathbf{p} \pm|\mathbf{p}| \sqrt{1+\Lambda^{2}}\right)^{2}},
$$

where we have defined the vector $\Lambda^{i}=\kappa^{0 i}$ and

$$
\mathcal{J}=(\mathbf{p} \times \mathbf{J})^{2}+\mathbf{J}^{2}\left[\Lambda^{2} \mathbf{p}^{2}-(\boldsymbol{\Lambda} \cdot \mathbf{p})^{2}\right]+2 p_{0}\left[(\boldsymbol{\Lambda} \cdot \mathbf{p}) \mathbf{J}^{2}-(\mathbf{p} \cdot \mathbf{J})(\boldsymbol{\Lambda} \cdot \mathbf{J})\right]
$$

which can be rewritten by choosing the following orthonormal basis composed by the vectors $\mathbf{u}, \mathbf{v}$ :

$$
\mathbf{u}=\frac{\mathbf{p}}{|\mathbf{p}|}, \quad \mathbf{v}=\frac{\boldsymbol{\Lambda}-(\boldsymbol{\Lambda} \cdot \mathbf{u}) \mathbf{u}}{|\boldsymbol{\Lambda}-(\boldsymbol{\Lambda} \cdot \mathbf{u}) \mathbf{u}|}, \quad \boldsymbol{\Lambda}=\left(\kappa_{01}, \kappa_{02}\right) .
$$

In this basis, we achieve

$$
\mathcal{J}=\left[J_{\mathbf{u}} \Lambda_{\mathbf{v}}|\mathbf{p}|-p_{0} J_{\mathbf{v}}\right]^{2}
$$

and we have $p=|\mathbf{p}| u, \quad \Lambda=\Lambda_{\mathbf{u}} u+\Lambda_{\mathbf{v}} v, \quad J=J_{\mathbf{u}} u+J_{\mathbf{v}} v$. Thus, the saturation is read

$$
S P=i\left[\frac{J_{\mathbf{u}} \Lambda_{\mathbf{v}}|\mathbf{p}|-p_{0} J_{\mathbf{v}}}{\boldsymbol{\Lambda} \cdot \mathbf{p} \pm|\mathbf{p}| \sqrt{1+\boldsymbol{\Lambda}^{2}}}\right]^{2}
$$

which is positive-definite, compatible with the unitarity requirements.

We finally consider the anisotropic configuration represented by the coefficients $\kappa_{i j}$. In this case, $\varrho=1-\operatorname{tr}\left(\kappa_{i j}\right)+$ $\operatorname{det}\left(\kappa_{i j}\right)$, and the saturation is

$$
S P=i \frac{\kappa_{i j} J_{i} J_{j}+\left[1-\operatorname{tr}\left(\kappa_{i j}\right)\right]\left[\mathbf{J}^{2}-\left(J_{0}\right)^{2}\right]}{1-\operatorname{tr}\left(\kappa_{i j}\right)+\operatorname{det}\left(\kappa_{i j}\right)} .
$$

The numerator can be written as

$$
\kappa_{i j} J_{i} J_{j}+\left[1-\operatorname{tr}\left(\kappa_{i j}\right)\right]\left[\mathbf{J}^{2}-\left(J_{0}\right)^{2}\right]=\frac{\left[\epsilon_{i j} p_{i} J_{j}+\epsilon_{k j} \kappa_{j i} J_{k} p_{i}\right]^{2}}{\left[\mathbf{p}^{2}-\left(\kappa_{i j} p_{i} p_{j}\right)\right]},
$$

so that the saturation finally read

$$
S P=i \frac{\left[\epsilon_{i j} p_{i} J_{j}+\epsilon_{k j} \kappa_{j i} J_{k} p_{i}\right]^{2}}{\left[\mathbf{p}^{2}-\kappa_{i j} p_{i} p_{j}\right]\left[1-\operatorname{tr}\left(\kappa_{i j}\right)+\operatorname{det}\left(\kappa_{i j}\right)\right]},
$$

which is positive-definite for sufficiently small values of the background $\kappa_{i j}$.

The residue in the double pole $p^{2}=0$ is proportional to $p_{\mu} p_{\nu}$, and due to current conservation $\left(p_{\mu} J^{\mu}=0\right)$, its saturation is zero. Thus, this is a nonphysical pole. Therefore, the unique physical pole is $\nabla(p)=0$, which yields unitary modes, as examined above. 


\section{ONE APPLICATION: BPS VORTEX CONFIGURATIONS}

In a recent work 32], it was demonstrated the existence of BPS uncharged vortex configurations in the context of the nonbirefringent and Lorentz-violating model of Ref. [17], supplemented by a Higgs sector. It was argued that the Lorentz-violating parameter modifies the profiles of the BPS vortex solutions yielding a compactlike behavior similar to the ones stemming from the k-field theories [36], 37]. The existence of vortices in the LIV framework of Ref. 32. implies an expectation for the observation of vortices in the context of the planar model developed here when properly coupled to the Higgs field. In the following we show that this is really the case.

The starting point in the investigation about vortex configurations is the planar Lagrangian (5), supplemented with the Higgs field, $\varphi$,

$$
\mathcal{L}_{1+2}=-\frac{1}{4} F_{\mu \nu} F^{\mu \nu}-\frac{1}{2} \kappa_{\nu \rho} F_{\lambda}{ }^{\nu} F^{\lambda \rho}+\frac{1}{2}[1-\eta] \partial_{\mu} \phi \partial^{\mu} \phi+\frac{1}{2} \kappa_{\nu \rho} \partial^{\nu} \phi \partial^{\rho} \phi+\left|\mathcal{D}_{\mu} \varphi\right|^{2}-U(|\varphi|, \phi),
$$

coupled to the gauge sector by the covariant derivative, $\left(\mathcal{D}_{\mu} \varphi\right)=\partial_{\mu} \varphi-i e A_{\mu} \varphi$, and endowed with a suitable potential, $U(|\varphi|, \phi)$, able to satisfy BPS conditions. The neutral scalar field $\phi$, stemming from the dimensional reduction, plays a role analogue to the auxiliary field $N$ inserted in the Maxwell-Chern-Simons-Higgs model [34, 35] in order to provide BPS vortex solutions.

In the analysis of Ref. [32], we set $\kappa_{0 i}=0$, which restrains our study only to uncharged vortex solutions. In such circumstance, one of the BPS equations of the full model states that

$$
A_{0}=\mp \phi=0,
$$

The condition $A_{0}=0$ is compatible with uncharged solutions, and satisfies the Gauss's law trivially. Under this BPS condition, the potential becomes

$$
U(|\varphi|)=\frac{1}{2(1-s)}\left(e v^{2}-e|\varphi|^{2}\right)^{2}
$$

where $v$ represents the vacuum expectation value of the Higgs field and $s=\kappa_{i i}=\kappa_{11}+\kappa_{22}$.

This way, for uncharged solutions, the only nonnull stationary equations of motion of the model (59) are the Ampere law

$$
\left(\epsilon_{a j}-\epsilon_{b j} \kappa_{a b}-\epsilon_{a b} \kappa_{j b}\right) \partial_{a} B-i e\left(\varphi^{*} \partial_{j} \varphi-\varphi \partial_{j} \varphi^{*}\right)-2 e^{2} A_{j}|\varphi|^{2}=0,
$$

and the equations of motion for the Higgs field,

$$
\nabla^{2} \varphi-2 i e A_{j} \partial_{j} \varphi+e^{2} A_{0}^{2} \varphi-e^{2} A_{j}^{2} \varphi-e^{2} \phi^{2} \varphi-\frac{\partial U(|\varphi|)}{\partial \varphi^{*}}=0 .
$$

In this stationary regime, the canonical energy of the uncharged system is written as

$$
E= \pm e v^{2} \int d^{2} x B+\int d^{2} x\left\{\frac{1}{2}(1-s)\left[B \mp \frac{e v^{2}-e|\varphi|^{2}}{(1-s)}\right]^{2}+\left|D_{ \pm} \varphi\right|^{2} \mp \frac{1}{2} \epsilon_{i j} \partial_{i} J_{j}\right\} .
$$

Here, we have defined the operators

$$
D_{ \pm} \varphi=D_{1} \varphi \pm i D_{2} \varphi
$$

and the current density $J_{j}=i\left(\varphi^{*} \partial_{j} \varphi-\varphi \partial_{j} \varphi^{*}\right)+2 e A_{j}|\varphi|^{2}$.

In order to minimize the energy, we impose other two BPS conditions

$$
\begin{aligned}
& D_{ \pm} \varphi=0 \\
& B= \pm \frac{e v^{2}-e|\varphi|^{2}}{(1-s)} .
\end{aligned}
$$


The three BPS conditions, (60), (66) and (67), reduce the energy to be proportional to the magnetic flux,

$$
E_{B P S}= \pm e v^{2} \int d^{2} x B
$$

once the term $\epsilon_{i j} \partial_{i} J_{j}$ does not contribute to the energy. It is worthwhile to note that the BPS equations reproduce the Ampere's law (62) and the stationary equation for the Higgs field (63). The vortex solutions are provided by the BPS equations, (66) and (67), under appropriated boundary conditions.

In order to search for uncharged stable vortex configurations, we state the usual ansatz for static rotationally symmetric vortex solutions, working in polar coordinates $(r, \theta)$, and wherein the fields are parametrized as

$$
A_{\theta}=-\frac{1}{e r}[a(r)-n], \quad \varphi=v g(r) e^{i n \theta},
$$

where $a(r), g(r)$ are regular scalar functions satisfying appropriated boundary conditions that provide topological vortex solutions possessing a finite total energy:

$$
\begin{gathered}
g(r \rightarrow \infty) \rightarrow 1, \quad a(r \rightarrow \infty) \rightarrow 0^{ \pm}, \\
g(r \rightarrow 0) \rightarrow 0, \quad a(r \rightarrow 0) \rightarrow n,
\end{gathered}
$$

where the signal + corresponds to $n>0$ and - to $n<0$, with $n$ being the winding number of the topological solution. In this ansatz the magnetic field is

$$
B(r)=-\frac{a^{\prime}}{e r}
$$

while the BPS equations, (66) and (67), are written as

$$
\begin{aligned}
g^{\prime} & = \pm \frac{a g}{r}, \\
\frac{a^{\prime}}{e r} & =\mp \frac{e v^{2}}{(1-s)}\left(1-g^{2}\right) .
\end{aligned}
$$

We note that these are the same BPS equations attained in Ref. [32], so that the same results can be achieved here by numerical simulations. We have thus shown that the present planar model supports uncharged BPS solutions with a compactlike profile. Although this first investigation has not unveiled a new BPS behavior in relation to the uncharged profiles of Ref. 32], it confirms that this planar model supports vortex solutions which could find application in planar systems where the spatial extension of the defect depends on some additional parameter.

Another interesting investigation is to analyze the existence of charged vortices in the planar Lorentz-violating model of Lagrangian (59) (in the absence of the Chern-Simons term), such possibility is supported by its Gauss's law

$$
\left(1+\kappa_{00}\right) \partial_{j} \partial_{j} A_{0}-\kappa_{i j} \partial_{i} \partial_{j} A_{0}+\epsilon_{i j} \kappa_{0 i} \partial_{j} B-2 e^{2} A_{0}|\phi|^{2}=0 .
$$

which explicitly shows that a nonnull $\kappa_{0 i}$ LIV parameter mixes the electric and magnetic sectors of the model, opening the possibility for existence of charged solutions.

\section{CONCLUSIONS}

In this work, we have continued examining the nonbirefringent CPT-even planar electrodynamics stemming from the dimensional reduction of the CPT-even gauge sector of the SME, first derived in Ref. [25]. More specifically, we have explicitly evaluated the Feynman propagator for this planar model and analyzed some of its properties. The Feynman's propagator of the electromagnetic sector was carried out using a set of ten tensors forming a closed algebra. In the end, the gauge propagator was expressed in terms of only eight of these tensors, arranged in such a way to yield five symmetric linear independent tensors. The propagator poles yielded dispersion relations which are compatible with the ones of Ref. 25] and revealed the existence of only one degree of freedom for each propagator, as 
it is usual in a (1+2)-dimensional electrodynamics. The causality and unitarity of electromagnetic and scalar sectors were examined. Such analysis has shown that the isotropic parity-even sector is stable and unitary for $\left|\kappa_{00}\right|<1$, but causal for $0 \leq \kappa_{00} \leq 1$. So, this sector is stable, causal and unitary for $0 \leq \kappa_{00}<1$. On the other hand, the anisotropic sector ruled by $\kappa_{0 i}$ is stable and unitary for arbitrary values of $\kappa_{0 i}$, however it can be noncausal for the some configurations of $\kappa_{0 i}$. Finally, the anisotropic sector characterized by $\kappa_{i j}$ is stable and unitary for sufficiently small values of the LIV coefficients, but it is in general noncausal. This behavior is similar with the scenario of the original (1+3)-dimensional theory [17] from which this planar model was derived.

Finally, we have shown that the planar model examined in this work can support uncharged BPS vortex solutions very similar to the ones of Ref. [32], which can find applications in condensed matter system where this Lorentzviolating electrodynamics may be interpreted as an effective theory describing, for example, anisotropies or impurities of a particular material. This first study also raises the issue about the existence of BPS planar charged vortex solutions (in the absence of the Chern-Simons terms). As one knows, the attainment of charged vortices is only possible when the Chern-Simons term is considered [33, 35], once this term couples the electric and magnetic sectors of the theory. In the present model, such coupling is played by the Lorentz-violating parameter $\kappa_{0 i}$, which implies the inclusion of the term $\epsilon_{i j} \kappa_{0 i} \partial_{j} B$ in the Gauss's law of the full model (75). With it, we achieve a richer model which certainly engenders charged planar BPS vortex configurations. This investigation is under finalization [38] and will be reported elsewhere.

\section{Acknowledgments}

The authors are grateful to FAPEMA, CAPES and CNPq (Brazilian research agencies) for invaluable financial support.

[1] V. A. Kostelecky and S. Samuel, Phys. Rev. Lett. 63, 224 (1989); Phys. Rev. Lett. 66, 1811 (1991); Phys. Rev. D 39, 683 (1989); Phys. Rev. D 40, 1886 (1989), V. A. Kostelecky and R. Potting, Nucl. Phys. B 359, 545 (1991); Phys. Lett. B 381, 89 (1996); V. A. Kostelecky and R. Potting, Phys. Rev. D 51, 3923 (1995).

[2] D. Colladay and V. A. Kostelecky, Phys. Rev. D 55, 6760 (1997); D. Colladay and V. A. Kostelecky, Phys. Rev. D 58, 116002 (1998); S. R. Coleman and S. L. Glashow, Phys. Rev. D 59, 116008 (1999); S.R. Coleman and S.L. Glashow, Phys. Rev. D 59, 116008 (1999).

[3] S.M. Carroll, G.B. Field and R. Jackiw, Phys. Rev. D 41, 1231 (1990).

[4] V. A. Kostelecky and M. Mewes, Phys. Rev. Lett. 87, 251304 (2001).

[5] V. A. Kostelecky and M. Mewes, Phys. Rev. D 66, 056005 (2002).

[6] V. A. Kostelecky and M. Mewes, Phys. Rev. Lett. 97, 140401 (2006).

[7] V. A. Kostelecky and M. Mewes, Phys. Rev. D 80, 015020 (2009).

[8] M.S. Berger, V. A. Kostelecky, Phys.Rev.D 65, 091701 (2002); H. Belich , J.L. Boldo, L.P. Colatto, J.A. Helayel-Neto, A.L.M.A. Nogueira, Phys.Rev. D 68, 065030 (2003); A.P. Baeta Scarpelli, H. Belich, J.L. Boldo, L.P. Colatto, J.A. Helayel-Neto, A.L.M.A. Nogueira, Nucl. Phys. Proc. Suppl.127, 105-109 (2004).

[9] R. Lehnert and R. Potting, Phys. Rev. Lett. 93, 110402 (2004); R. Lehnert and R. Potting, Phys. Rev. D 70, 125010 (2004); B. Altschul, Phys. Rev. D 75, 105003 (2007); C. Kaufhold and F.R. Klinkhamer, Nucl. Phys. B 734, 1 (2006).

[10] B. Altschul, Nucl. Phys. B 796, 262 (2008); B. Altschul, Phys. Rev. Lett. 98, 041603 (2007); C. Kaufhold and F.R. Klinkhamer, Phys. Rev. D 76, 025024 (2007).

[11] R. Jackiw and V. A. Kostelecký, Phys. Rev. Lett. 82, 3572 (1999); J. M. Chung and B. K. Chung Phys. Rev. D 63, 105015 (2001); J.M. Chung, Phys.Rev. D 60, 127901 (1999); G. Bonneau, Nucl.Phys. B 593, 398 (2001); M. Perez-Victoria, Phys. Rev. Lett. 83, 2518 (1999); M. Perez-Victoria, J. High. Energy Phys. 0104, (2001) 032; A. P. B. Scarpelli, M. Sampaio, M. C. Nemes, and B. Hiller, Phys. Rev. D 64, 046013 (2001); T. Mariz, J.R. Nascimento, E. Passos, R.F. Ribeiro and F.A. Brito, J. High. Energy Phys. 0510 (2005) 019; J. R. Nascimento, E. Passos, A. Yu. Petrov, F. A. Brito, J. High. Energy Phys. 0706, (2007) 016; B. Altschul, Phys. Rev. D 70, 101701 (2004); A.P.B. Scarpelli, M. Sampaio, M.C. Nemes, B. Hiller, Eur. Phys. J. C 56, 571 (2008); G. Gazzola, H. G. Fargnoli, A. P. Baêta Scarpelli, Marcos Sampaio, M. C. Nemes, J. Phys. G 39, 035002 (2012). 
[12] M. Frank and I. Turan, Phys. Rev. D 74, 033016 (2006); O.G. Kharlanov, V.Ch. Zhukovsky, Phys. Rev. D81, 025015 (2010).

[13] J.-Q. Xia, Hong Li, X. Wang, X. Zhang, Astron.Astrophys. 483, 715 (2008); J.-Q. Xia, H. Li, X. Zhang, Phys.Lett. B 687, 129(2010); B. Feng, M. Li, J.-Q. Xia, X. Chen, X. Zhang, Phys. Rev. Lett. 96, 221302 (2006); P. Cabella, P. Natoli, J. Silk, Phys. Rev. D 76, 123014 (2007).

[14] M. Gomes, J. R. Nascimento, A. Yu. Petrov, A. J. da Silva, Phys. Rev. D 81, 045018 (2010); M. Gomes, T. Mariz, J. R. Nascimento, A. Yu. Petrov, A. F. Santos, A. J. da Silva, Phys.Rev.D 81, 045013 (2010).

[15] C. Adam and F. R. Klinkhamer, Nucl. Phys. B 607, 247 (2001); C. Adam and F. R. Klinkhamer, Nucl. Phys. B 657, 214 (2003); A. P. Baeta Scarpelli, H. Belich, J. L. Boldo, J.A. Helayel-Neto, Phys. Rev. D 67, 085021 (2003).

[16] A.A. Andrianov and R. Soldati, Phys. Rev. D 51, 5961 (1995); Phys. Lett. B 435, 449 (1998); A.A. Andrianov, R. Soldati and L. Sorbo, Phys. Rev. D 59, 025002 (1998); A. A. Andrianov, D. Espriu, P. Giacconi, R. Soldati, J. High Energy Phys. 0909, 057 (2009); J. Alfaro, A.A. Andrianov, M. Cambiaso, P. Giacconi, R. Soldati, Int.J.Mod.Phys. A 25, 3271 (2010); V. Ch. Zhukovsky, A. E. Lobanov, E. M. Murchikova, Phys.Rev. D 73 065016, (2006).

[17] R. Casana, M. M. Ferreira Jr., A.R. Gomes, F. E. P. dos Santos, Phys.Rev. D 82, 125006 (2010).

[18] F.R. Klinkhamer, M. Schreck, Nucl. Phys. B 848, 90 (2011).

[19] F.R. Klinkhamer and M. Risse, Phys. Rev. D 77, 016002 (2008); F.R. Klinkhamer and M. Risse, Phys. Rev. D 77, 117901 (A) (2008).

[20] F. R. Klinkhamer and M. Schreck, Phys. Rev. D 78, 085026 (2008).

[21] V. A. Kostelecky and A.G.M. Pickering, Phys. Rev. Lett. 91, 031801 (2003); B. Altschul, Phys.Rev. D 70, 056005 (2004).

[22] C.D. Carone, M. Sher, and M. Vanderhaeghen, Phys. Rev. D 74, 077901 (2006); B. Altschul, Phys. Rev. D 79, 016004 (2009).

[23] M.A. Hohensee, R. Lehnert, D. F. Phillips, R. L. Walsworth, Phys. Rev. D 80, 036010(2009); M.A. Hohensee, R. Lehnert, D. F. Phillips, R. L. Walsworth, Phys. Rev. Lett. 102, 170402 (2009); B. Altschul, Phys. Rev. D 80, 091901(R) (2009).

[24] R. Casana, E.S. Carvalho, M. M. Ferreira Jr., Phys. Rev. D 84, 045008 (2011).

[25] R. Casana, M. M. Ferreira Jr., R. P. Machado Moreira, Phys. Rev. D 84, 125014 (2011).

[26] M.N. Barreto, D. Bazeia, R. Menezes, Phys. Rev. D 73, 065015 (2006); A. de Souza Dutra, M. Hott, and F. A. Barone, Phys. Rev. D 74, 085030 (2006); A. de Souza Dutra, R. A. C. Correa, Phys. Rev. D 83, 105007 (2011).

[27] M. A. Anacleto, F. A. Brito, E. Passos, Phys. Lett. B 694, 149 (2010); M. A. Anacleto, F. A. Brito, E. Passos, Phys. Lett. B 703, 609 (2011); Phys. Rev. D 85, 025013 (2012).

[28] R. Casana and K. A. T. da Silva, arXiv:1106.5534.

[29] R. U. Sexl and H.K. Urbantke, "Relativity, Groups, Particles: special relativity and relativistic symmetry in field and particle physics", Springer-Verlag, New York (1992).

[30] M. Veltman, "Quantum Theory of Gravitation", in Methods in Field Theory, edited by R. Bailian and J. Zinn-Justin (North-Holland Publising Company and World Scientific Publising Co Ltd, Singapore, 1981).

[31] S. Deser, R. Jackiw, and S. Templeton, Ann. Phys. (NY) 140, 372 (1982).

[32] C. M. Cantanhede, R. Casana, M. M. Ferreira Jr., E. da Hora, arXiv:1109.2931

[33] R. Jackiw and E. J. Weinberg, Phys. Rev. Lett. 64, 2234 (1990); R. Jackiw, K. Lee, and E.J. Weinberg, Phys. Rev. D 42, 3488 (1990); G.V. Dunne, Self-Dual Chern-Simons Theories (Springer, Heidelberg, 1995).

[34] J. Hong, Y. Kim, and P.Y. Pac, Phys. Rev. Lett. 64, 2230 (1990).

[35] S. Bolognesi and S.B. Gudnason, Nucl. Phys. B 805, 104 (2008).

[36] C. dos Santos, Phys. Rev. D 82, 125009 (2010); D. Bazeia, E. da Hora, R. Menezes, H. P. de Oliveira, and C. dos Santos, Phys. Rev. D 81, 125016 (2010); D. Bazeia, E. da Hora, C. dos Santos, R. Menezes, Eur. Phys. J. C 71, 1833 (2011).

[37] E. Babichev, Phys. Rev. D 74, 085004 (2006); Phys. Rev. D 77, 065021 (2008).

[38] C. M. Cantanhede, R. Casana, M. M. Ferreira Jr., E. da Hora, work under development. 